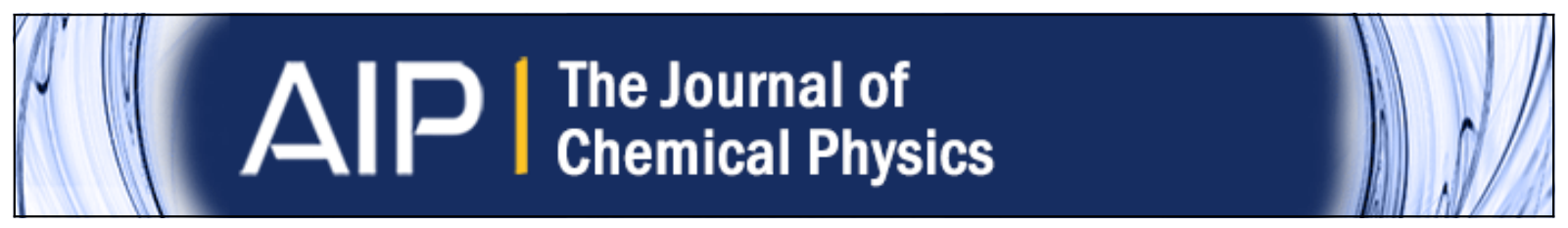

Exciton dissociation at donor-acceptor heterojunctions: Dynamics using the collective effective mode representation of the spin-boson model

Aurélie Chenel, Etienne Mangaud, Irene Burghardt, Christoph Meier, and Michèle Desouter-Lecomte

Citation: The Journal of Chemical Physics 140, 044104 (2014); doi: 10.1063/1.4861853

View online: http://dx.doi.org/10.1063/1.4861853

View Table of Contents: http://scitation.aip.org/content/aip/journal/jcp/140/4?ver=pdfcov

Published by the AIP Publishing

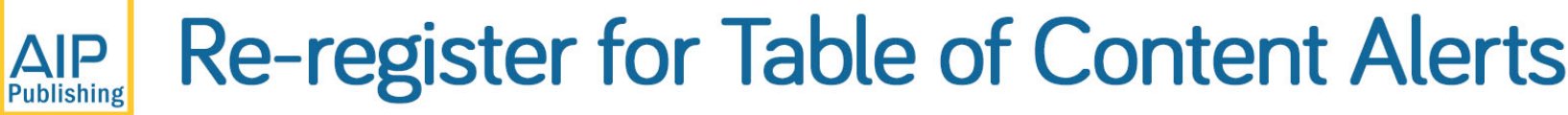

\section{Create a profile.




\title{
Exciton dissociation at donor-acceptor heterojunctions: Dynamics using the collective effective mode representation of the spin-boson model
}

\author{
Aurélie Chenel, ${ }^{1,2}$ Etienne Mangaud, ${ }^{1,2}$ Irene Burghardt,, ${ }^{3, a)}$ Christoph Meier,, a) \\ and Michèle Desouter-Lecomte ${ }^{1,4, a)}$ \\ ${ }^{1}$ Laboratoire de Chimie Physique, Bât 349, Université Paris-Sud, UMR 8000, F-91405 Orsay, France \\ ${ }^{2}$ Laboratoire Collisions, Agrégats, Réactivité, UMR 5589, IRSAMC, Université Paul Sabatier, \\ F-31062 Toulouse, France \\ ${ }^{3}$ Institut für Physikalische und Theoretische Chemie, Goethe Universität Frankfurt, Max-von-Laue-Str. 7, \\ D-60438 Frankfurt am Main, Germany \\ ${ }^{4}$ Département de Chimie, Université de Liège, Sart Tilman, B6, B-4000 Liège, Belgium
}

(Received 19 September 2013; accepted 30 December 2013; published online 23 January 2014)

Following the recent quantum dynamics investigation of the charge transfer at an oligothiophenefullerene heterojunction by the multi-configuration time dependent Hartree method [H. Tamura, R. Martinazzo, M. Ruckenbauer and I. Burghardt, J. Chem. Phys. 137, 22A540 (2012)], we revisit the transfer process by a perturbative non-Markovian master equation treated by the time local auxiliary density matrix approach. We compare the efficiency of the spin-boson model calibrated by quantum chemistry with the effective mode representation. A collective mode is extracted from the spin-boson spectral density. It is weakly coupled to a residual bath of vibrational modes, allowing second-order dynamics. The electron transfer is analyzed for a sampling of inter-fragment distances showing the fine interplay of the electronic coupling and energy gap on the relaxation. The electronic coherence, expected to play a role in the process, is preserved during about 200 fs. @ 2014 AIP Publishing LLC. [http://dx.doi.org/10.1063/1.4861853]

\section{INTRODUCTION}

The exciton dissociation dynamics of an oligothiophene $\left(\mathrm{OT}_{4}\right) /$ fullerene $\left(\mathrm{C}_{60}\right)$ donor-acceptor complex has been recently investigated ${ }^{1,2}$ as a model for bulk heterojunctions of conjugated polymers and fullerene (poly-3hexylthiophene (P3HT) and phenyl-C61 butyric acid methyl ester (PCBM)). ${ }^{3,4}$ Exciton dissociation is a basic mechanism in the energy conversion in organic photovoltaic cells and typically occurs within a timescale of a few hundreds of femtoseconds. A spin-boson type Hamiltonian was parametrized by LC-TDDFT calculations (time-dependent DFT with longrange correction ${ }^{5}$ ), which provided electronic energies, couplings, and the spectral density. The latter is the key data in dissipative dynamics. It specifies the coupling between the system and the environment as a function of the bath frequency. When the surrounding contains some hundreds of modes, it can be seen as a micro-environment, permitting an explicit calculation of the unitary system-bath wavefunction evolution by the MCTDH (Multi Configuration Time Dependent Hartree $)^{6,7}$ method and an approximate model Hamiltonian. ${ }^{1,2,8-11}$ The discretization of the oscillator bath leads to a finite Poincaré recurrence time but, if the latter is long enough, correct information about the early relaxation can be obtained.

In this work, we use the system described above to investigate the efficiency of dissipative non-Markovian quantum dynamics with an oscillator bath treated as a continuum. In

\footnotetext{
a)E-mail: michele.desouter-lecomte@u-psud.fr, chris@irsamc.ups-tlse.fr, and burghardt@chemie.uni-frankfurt.de
}

a first approach, the inter-fragment $\left(\mathrm{OT}_{4}\right.$-fullerene) distance $(R)$, which has an important effect on the electronic coupling, is not taken as a dynamical coordinate and the transfer is studied by sampling different values of $R$. We first investigate the crude spin-boson model by a second-order non-Markovian master equation ${ }^{12,13}$ with the time local auxiliary density matrix method. ${ }^{14,15}$ This approach is a time-convoluted secondorder quantum master equation also known as the generalized Bloch-Redfield equations. They have been compared with different approximations in a benchmark study ${ }^{16}$ and recently shown to correctly describe the energy transfer in lightharvesting sytems. ${ }^{17}$ Here, the spectral density is fitted by a sum of Lorentzian functions. ${ }^{14,15} \mathrm{~A}$ similar approach has been applied in the context of laser control ${ }^{18,19}$ and adapted in a time local approach. ${ }^{20-22}$ Obviously, the second order has limitation and for some inter-fragment distances the approximation dramatically fails. The reduced hierarchy equations of motion (HEOM) ${ }^{23-25}$ or the recent interesting alternative combining stochastic sampling with the hierarchy equations $(\mathrm{sHEOM})^{26}$ go beyond the second order and have been used in different applications. ${ }^{27-31}$ Further, regarding the Lorentzian fitting mentioned above, ${ }^{14,15}$ a different parametrization could be advantageous, based on a hierarchical continued-fraction expansion of the spectral density. ${ }^{32}$ This strategy, in conjunction with HEOM, is left for a future complementary work. ${ }^{33}$

While the present work remains in the second-order framework and keeps the conventional Lorentzian fitting of the spectral density, we circumvent the limitation of weak system-bath coupling by going to the Brownian oscillator representation of the spin-boson model. In the spin-boson Hamiltonian the system is formed by the electronic states only while 
all the nuclear vibrators belong to the surrounding bath. It can be recast by a canonical transformation of the oscillators to include the main collective mode in the system and treat the remaining modes as a perturbative secondary bath which interacts only indirectly with the electronic states. ${ }^{34-41}$ By this way, it is expected that the second-order treatment can be justified. The idea of the canonical transformation has been recently revisited in the context of photophysics involving conical intersections ${ }^{42,43}$ for which three effective modes are necessary. A hierarchical chain of nearest-neighbor coupled modes, and the residual spectral density resulting from a truncation of the chain at a given order, have been derived. ${ }^{10,44-46}$

\section{MODEL AND METHODS}

The TDDFT investigation in Ref. 1 has provided the electronic structure of the supermolecule oligothiophene $\left(\mathrm{OT}_{4}\right)$ fullerene $\left(\mathrm{C}_{60}\right)$ for different values of the inter-fragment distance $R$ (see Fig. 1). We focus on two diabatic states (see Refs. 1 and 47 for details on the diabatization procedure): a photoinduced excitonic donor state $\mathrm{OT}_{4}{ }^{*}-\mathrm{C}_{60}$ denoted $\mathrm{XT}$ and a charge separated state $\mathrm{OT}_{4}^{+}-C_{60}^{-}$denoted CT.

Figure 2 gives a schematic illustration of the two alternative dynamical models we use. In the spin-boson model the system only contains the two XT and CT diabatic electronic states at their equilibrium geometry. The energy gap $\Delta_{\mathrm{XT}-\mathrm{CT}}$ and the electronic coupling $V_{\mathrm{XT}-\mathrm{CT}}$ depend on the inter-fragment distance $R$. $V_{\mathrm{XT}-\mathrm{CT}}$ is assumed to be independent of the other intramolecular vibrations. The values used in the simulations are given in Table I. The Rabi period of the isolated system and the weights of the diabatic states in the ground adiabatic state are also given to discuss the relaxation process. The bath is formed by all the intramolecular modes of the two moieties (with a total number of modes $N$ $=264$ ). The main approximations are the harmonic approximation and the assumption that the normal modes are similar in both electronic states but their equilibrium positions are shifted. These displacements of the equilibrium positions lead

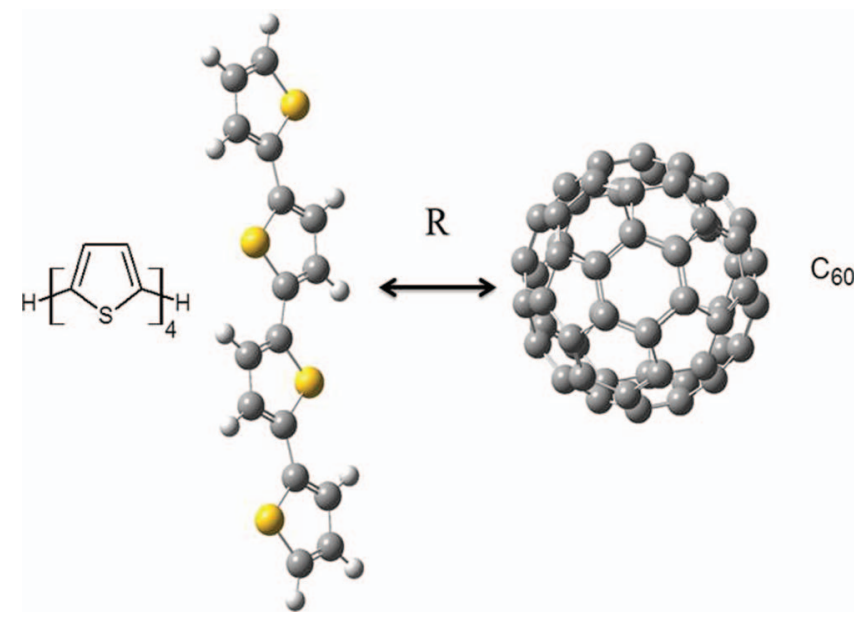

FIG. 1. Schematic representation of the supermolecule oligothiophene $\left(\mathrm{OT}_{4}\right)$-fullerene $\left(\mathrm{C}_{60}\right)$ for which the inter-fragment distance $R$ is not taken as a dynamical variable in the simulations. In the chosen stack structure a 6 member ring of $\mathrm{C}_{60}$ faces the $\mathrm{CC}$ bond between thiophene rings (stack denoted 1 in Ref. 1).

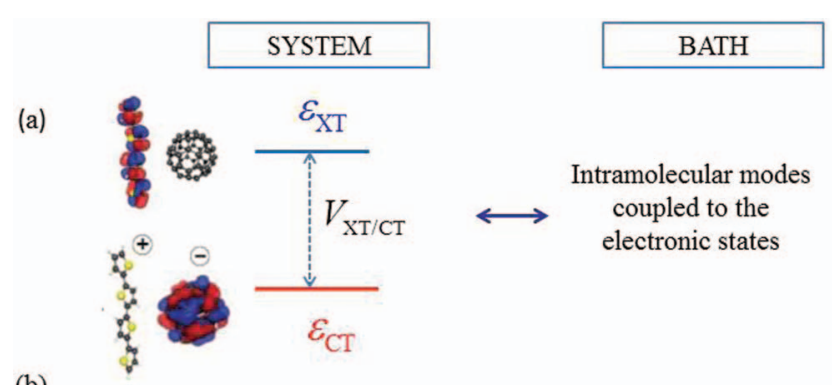

(b)

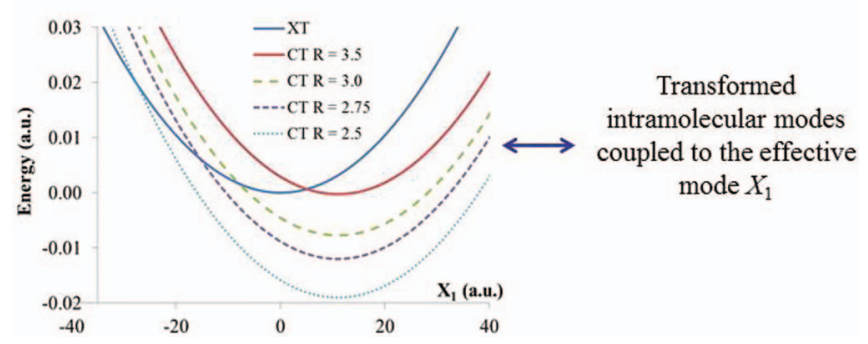

FIG. 2. Schematic representation of the two models of dissipative dynamics for the charge transfer at the oligothiophene-fullerene heterojunction: (a) spin-boson model and (b) effective mode representation of the spin-boson. The $V_{X T}$ and $V_{C T}$ potential energy curves are drawn for different interfragment distances $R$ in $\AA$.

to vibronic couplings and are the main quantities defining the spectral density $J_{0}(\omega)$ discussed below. In the effective-mode representation of the spin-boson model, the energy gap variation with the effective mode $X_{1}$ is taken into account. The other modes are not directly coupled to the electronic states but only to $X_{1}$ with a spectral density $J_{1}(\omega)$. Another approach based on a Taylor expansion of a linear reaction path interpolating between reactive and product could also be probed. ${ }^{48}$

\section{A. Auxiliary density matrix formalism}

We summarize here the main dissipative equations at the second order for a generic system-bath partitioning. The equations will be made more explicit below for the two partitionings described above: the spin-boson case versus the effective mode representation. The reduced density matrix for the system is obtained by tracing over the bath modes. It takes the following form, under the assumption that the full density matrix is separable initially: ${ }^{14}$

$$
\dot{\rho}_{S}(t)=L_{e f f} \rho_{S}(t)+\int_{0}^{t} d \tau K(t, \tau) \rho_{S}(\tau),
$$

where $L_{e f f}$ stands for $(-i / \hbar)\left[H_{e f f},.\right]$ and the effective Hamiltonian includes the renormalization term $H_{\text {eff }}=H_{S}+H_{\text {ren }}$. The

TABLE I. Parameters of the spin-boson model for different values of the inter-fragment distance $R \cdot\left|C_{\mathrm{XT}}\right|^{2}$ and $\left|C_{\mathrm{CT}}\right|^{2}$ are the weights of the diabatic $\mathrm{XT}$ and $\mathrm{CT}$ states in the ground adiabatic state.

\begin{tabular}{lccccc}
\hline \hline$R(\AA)$ & $\Delta_{\text {ХT-СT }}(\mathrm{eV})$ & $V_{\text {XT-CT }}(\mathrm{eV})$ & $\tau_{\text {Rabi }}(\mathrm{fs})$ & $\left|C_{\mathrm{XT}}\right|^{2}$ & $\left|C_{\mathrm{CT}}\right|^{2}$ \\
\hline 2.50 & 0.517 & 0.200 & 6.3 & 0.105 & 0.895 \\
2.75 & 0.327 & 0.185 & 8.3 & 0.169 & 0.831 \\
3.00 & 0.210 & 0.130 & 12.3 & 0.186 & 0.814 \\
3.25 & 0.130 & 0.098 & 17.5 & 0.223 & 0.777 \\
3.50 & 0.007 & 0.070 & 29.4 & 0.475 & 0.525 \\
\hline \hline
\end{tabular}


memory kernel at the second order becomes

$$
K(t-\tau) \rho_{S}(\tau)=\operatorname{Tr}_{B}\left(L_{S B} e^{\left(L_{B}+L_{S}\right)(t-\tau)} L_{S B} \rho_{B}\right) \rho_{S}(\tau),
$$

where $L_{S B}=(-i / \hbar)\left[H_{S B},.\right]$ involves the system-bath coupling and $\rho_{B}$ is the bath density matrix at Boltzmann equilibrium. The corresponding two-time correlation function is linked to the spectral density by the relation:

$$
C(t-\tau)=\frac{1}{\pi} \int_{-\infty}^{\infty} J(\omega) n_{\beta}(\omega) e^{i \omega(t-\tau)} d \omega,
$$

with $n_{\beta}(\omega)=1 /\left(e^{\beta \omega}-1\right)$ being the Bose function. Assuming that the spectral density $J(\omega)$ can be fitted by Lorentzian functions: ${ }^{14}$

$$
\begin{aligned}
J(\omega) & =\sum_{k=1}^{m} j_{k}(\omega) \\
& =\sum_{k=1}^{m} p_{k} \frac{\omega}{\left[\left(\omega+\Omega_{k}\right)^{2}+\Gamma_{k}^{2}\right]\left[\left(\omega-\Omega_{k}\right)^{2}+\Gamma_{k}^{2}\right]},
\end{aligned}
$$

the correlation function can be recast as a sum of decreasing complex exponentials,

$$
C(t-\tau)=\sum_{k=1}^{n} \alpha_{k} e^{i \gamma_{k}(t-\tau)}
$$

Here, $2 m$ terms stem from the poles of $J(\omega)$, namely

$$
\begin{aligned}
& \alpha_{2 l-1}=\frac{p_{l}}{8 \Omega_{l} \Gamma_{l}}\left[\operatorname{coth}\left(\beta\left(\Omega_{l} \pm i \Gamma_{l}\right) / 2\right) \mp 1\right] \text { and } \\
& \gamma_{2 l-1}= \pm \Omega_{l}+i \Gamma_{l} \quad \text { for } l=1, \ldots m
\end{aligned}
$$

and the other terms stem from the poles of the Bose function $\alpha_{k}=2 i J_{0}\left(i v_{k}\right) / \beta, \gamma_{k}=i v_{k}$ for $k>2 m$ where $v_{k}=2 \pi(k-2 m) /$ $\beta$ are the Matsubara frequencies.

\section{B. Spin-boson model}

The Hamiltonian of the electronic diabatic states reads

$$
H_{S}=\left(\begin{array}{cc}
\varepsilon_{\mathrm{XT}} & V_{\mathrm{XT}-\mathrm{CT}} \\
V_{\mathrm{XT}-\mathrm{CT}} & \varepsilon_{\mathrm{CT}}
\end{array}\right) \text {. }
$$

The Hamiltonians of the harmonic baths in massweighted coordinates are

$$
\begin{aligned}
H_{\mathrm{XT}}^{B} & =\sum_{i} \frac{1}{2}\left(p_{i}^{2}+\omega_{i}^{2} q_{i}^{2}\right), \\
H_{\mathrm{CT}}^{B} & =\sum_{i} \frac{1}{2}\left(p_{i}^{2}+\omega_{i}^{2}\left(q_{i}-d_{i}\right)^{2}\right) \\
& =\sum_{i} \frac{1}{2}\left(p_{i}^{2}+\omega_{i}^{2} q_{i}^{2}\right)-\sum_{i} \omega_{i}^{2} d_{i} q_{i}+\sum_{i} \frac{\omega_{i}^{2} d_{i}^{2}}{2}
\end{aligned}
$$

where $d_{i}$ is the displacement of the equilibrium position of the $i$ th CT normal mode with respect to that of the XT mode taken as a reference geometry. The validity of the harmonic model can be verified by comparing the renormalization energy given by $\lambda_{\mathrm{CT}}=H_{\text {ren }}=\sum_{i} \frac{\omega_{i}^{2} d_{i}^{2}}{2}=0.135 \mathrm{eV}$ with the value $(\sim 0.13 \mathrm{eV})$ obtained by quantum chemistry geometry optimization. ${ }^{1}$ The bilinear coupling between the system and the bath arises from the term $H_{S B}=-\sum_{i} c_{i} q_{i}|\mathrm{CT}\rangle\langle\mathrm{CT}|$ with $c_{i}=\omega_{i}^{2} d_{i}$. The discrete spectral density is built from the potential shifts $d_{i}$ of the $\mathrm{OT}_{4}{ }^{+}$and $\mathrm{C}_{60}{ }^{-}$fragments, discarding the intermolecular distance $R$. As shown in Fig. 4 of Ref. 1 , the frequency range covers the range up to $1700 \mathrm{~cm}^{-1}$ with some weak contributions around $3200 \mathrm{~cm}^{-1}$. The highest peaks in the region $1600-1700 \mathrm{~cm}^{-1}$ correspond to CS and $\mathrm{CC}$ stretching modes and those at low frequencies below $800 \mathrm{~cm}^{-1}$ are due to $\mathrm{OT}_{4}{ }^{+}$in-plane bending modes and to radial fullerene $\mathrm{H}_{\mathrm{g}}$ modes. A few weak peaks around $3200 \mathrm{~cm}^{-1}$ correspond to $\mathrm{CH}$ stretching in $\mathrm{OT}_{4}{ }^{+}$. The discrete spectral density is transformed to a continuous distribution by convoluting with a broadening Lorentzian function as done in Ref. 2:

$$
J_{0}(\omega)=\frac{\pi}{2} \sum_{k} \frac{c_{k}^{2}}{\omega_{k}} \delta\left(\omega-\omega_{k}\right) \approx \frac{\pi}{2} \sum_{k} \frac{c_{k}^{2}}{\omega_{k}} \frac{1}{\pi} \frac{\Delta}{\left(\omega-\omega_{k}\right)^{2}+\Delta^{2}},
$$

where $\Delta$ has been fixed here at the root-mean-square of the frequency spacing. This procedure with the chosen $\Delta$ value extends the density beyond $1700 \mathrm{~cm}^{-1}$. However, as shown in Ref. 2, the early dynamics before 50 fs remains completely unaffected and it has very little influence on the relevant timescale. Similarly, the peak around $3200 \mathrm{~cm}^{-1}$ can be disregarded because it does not modify the results obtained in the spin-boson approach. Its role in the dynamics after projecting an effective mode will be discussed below. $J_{0}(\omega)$ fitted by five Lorentzian functions [Eq. (4)] is shown in Fig. 3.
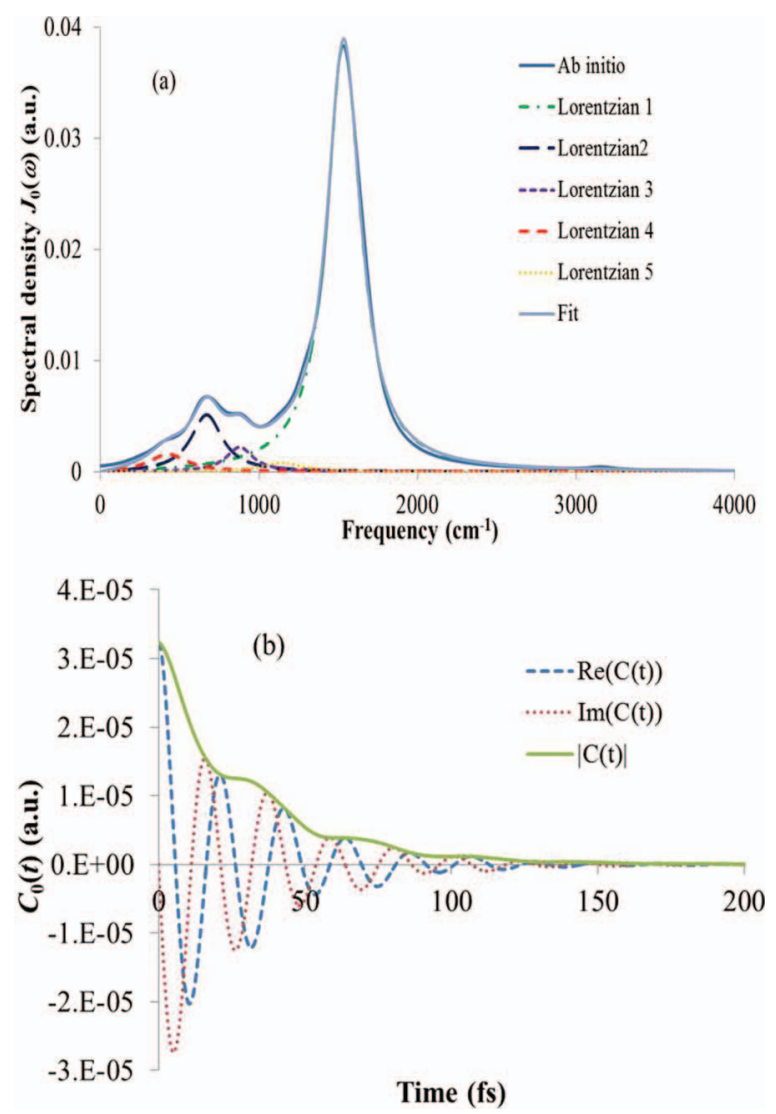

FIG. 3. (a) Fit of the convoluted ab initio spectral density $J_{0}(\omega)$ [Eq. (8)] by Lorentzian functions [Eq. (4)]. (b) Corresponding bath correlation function [Eq. (5)] at $T=300 \mathrm{~K}$. 
The corresponding parameters are given in the supplementary material. ${ }^{49}$

The operational expression of the memory kernel in the present spin-boson case becomes by inserting the parameterized form of $C_{0}(t-\tau)$ [Eq. (5)]:

$$
\begin{aligned}
& K(t-\tau) \rho_{S}(\tau) \\
& =i\left[|C T\rangle\langle C T|,\left\{i \sum_{k=1}^{n} \alpha_{0, k} e^{i \gamma_{0, k}(t-\tau)} e^{L_{s}(t-\tau)}|C T\rangle\right.\right. \\
& \left.\left.\quad \times\langle C T| \rho_{S}(\tau)\right\}+\{\text { h.c. }\}\right],
\end{aligned}
$$

where h.c. denotes the Hermitian conjugate. The integrodifferential equation [Eq. (1)] is replaced by a time local system of $(n+1)$ coupled equations where the auxiliary matrices are defined by

$$
\rho_{0, k}(t)=i \alpha_{0, k} \int_{0}^{t} d \tau e^{i \gamma_{0, k}(t-\tau)} e^{L_{S}(t-\tau)}|\mathrm{CT}\rangle\langle\mathrm{CT}| \rho_{S}(\tau) .
$$

The resulting coupled equations are

$$
\dot{\rho}_{S}(t)=L_{e f f} \rho_{S}(t)+i \sum_{k}\left[|\mathrm{CT}\rangle\langle\mathrm{CT}|, \rho_{0, k}(t)+\rho_{0, k}^{\dagger}(t)\right],
$$

$\dot{\rho}_{0, k}(t)=\left(L_{S}+i \gamma_{0, k}\right) \rho_{0, k}(t)+i \alpha_{0, k}|\mathrm{CT}\rangle\langle\mathrm{CT}| \rho_{S}(t) \quad k=1, n$.

The equations are solved by the fourth-order Runge Kutta $\operatorname{algorithm}^{50}$ or by the Split Operator method with a Cayley recursion $^{50}$ as suggested in Ref. 14.

\section{Effective mode representation}

Starting from the spin-boson Hamiltonian, a canonical transformation of the normal modes $\left\{q_{i}\right\} i=1, N \Rightarrow$ $\left\{X_{1}, x_{j}\right\} j=2, N$ can be performed. It allows one to define a single collective coordinate $X_{1}=\sum_{i=1}^{N} c_{i} q_{i} / D_{0}$ with $D_{0}^{2}$ $=\sum_{i=1}^{N} c_{i}^{2}$ coupled to the electronic system while the $N-1$ remaining modes are only coupled to this vibronic active mode and thus interact only indirectly with the electronic states. $^{16,32,34,36,37,39,41,45}$ The transformation can be chosen so that the secondary modes are uncoupled, i.e., so that the transformed Hessian matrix is block diagonal with diagonal elements $\Omega_{j}^{2}(j=2, N)$. The first diagonal element gives the effective frequency $\Omega_{1}^{2}$ and the first row or column contains the residual coupling $\bar{c}_{1 j}$. In mass-weighted coordinates, the system Hamiltonian becomes:

$$
\bar{H}_{S}=\left(\begin{array}{cc}
\varepsilon_{\mathrm{XT}}+\frac{P^{2}}{2}+\frac{\Omega_{1}^{2}}{2} X_{1}^{2} & V_{\mathrm{XT}-\mathrm{CT}} \\
V_{\mathrm{XT}-\mathrm{CT}} & \varepsilon_{\mathrm{CT}}+\frac{P^{2}}{2}+\frac{\Omega_{1}^{2}}{2}\left(X_{1}-\frac{D_{0}}{\Omega_{1}^{2}}\right)^{2}
\end{array}\right) .
$$

The transformed bath Hamiltonian now leads to a system-bath coupling $\bar{H}_{S B}=-\sum_{j=2}^{N} \bar{c}_{1 j} \mathbf{X}_{1} x_{j}$ where $\mathbf{X}_{1}$ is the matrix of the operator $X_{1}(|\mathrm{XT}\rangle\langle\mathrm{XT}|+| \mathrm{CT}\rangle\langle\mathrm{CT}|)$.

The discrete distribution of the new coupling elements $\bar{c}_{1 j}$ and the corresponding spectral density $J_{1}(\omega)$ can be obtained by building the transformation matrix from the eigenvectors associated with non-zero eigenvalues of the projected
Hessian $\mathbf{W}=\mathbf{P K P}+\mathbf{Q K Q}$ where $\mathbf{P}=\left|X_{1}\right\rangle\left\langle X_{1}\right|$ is the projector on the effective mode and $\mathbf{Q}=1-\mathbf{P} .^{41}$ On the other hand, the coupling, the frequency of the effective mode and the spectral density $J_{1}(\omega)$ can also be derived directly from the continuous approach by using $J_{0}(\omega):{ }^{45}$

$$
\begin{gathered}
D_{0}^{2}=\frac{2}{\pi} \int J_{0}(\omega) \omega d \omega, \\
\Omega_{1}^{2}=\frac{2}{\pi D_{0}^{2}} \int J_{0}(\omega) \omega^{3} d \omega .
\end{gathered}
$$

One obtains $D_{0}=5.74 \times 10^{-4}$ a.u. and $\Omega_{1}=1585 \mathrm{~cm}^{-1}$ by imposing a cutoff above $3000 \mathrm{~cm}^{-1}$ (see Fig. 3). In Ref. 45, a sequence of effective nearest-neighbor modes and the corresponding residual spectral densities are derived from an iterative procedure. Here we stop the chain after the first effective mode $X_{1}$. The residual spectral density is then given by

$$
J_{1}(\omega)=\frac{D_{0}^{2} J_{0}(\omega)}{\left|W_{0}^{+}(\omega)\right|^{2}}
$$

with $W_{0}^{+}(\omega)=\lim _{\varepsilon \rightarrow 0^{+}} \frac{1}{\pi} \int_{-\infty}^{\infty} \frac{J_{0}\left(\omega^{\prime}\right)}{\omega^{\prime}-(\omega+i \varepsilon)} d \omega^{\prime}$.

The function $\left|W_{0}^{+}(\omega)\right|^{2}=\left(\frac{1}{\pi} \sum_{k=1}^{m} P V \int_{-\infty}^{\infty} \frac{j_{k}\left(\omega^{\prime}\right)}{\omega^{\prime}-\omega} d \omega^{\prime}\right)^{2}$ $+\left(\sum_{k=1}^{m} j_{k}(\omega)\right)^{2}$, where $P V$ designates the Cauchy principal value, can be performed analytically when using the parametrization of Eq. (4). It is well known since the seminal work of Ref. 34 that when the spectral density of the spin-boson model is a single Lorentzian function $j_{k}(\omega)$, the spectral density of the effective mode model resulting from the canonical transformation is an Ohmic function linear in the frequency $J_{\text {ohmic }}=\eta \omega$ where $\eta=2 \Gamma_{k}$ is the friction of the Brownian coordinate. When several Lorentzian functions are summed, the main $j_{k}(\omega)$ fixes the background slope of $J_{1}(\omega)$ but secondary peaks are superimposed. Very weak peaks at high frequencies may be particularly enhanced. Note that the same amplifying effect is obtained by the discrete treatment which transforms the Hessian matrix. We have computed $J_{1}(\omega)$ by including or not the weak peak at $3100 \mathrm{~cm}^{-1}$ in $J_{0}(\omega)$. The result taking into account this peak and forcing a Lorentzian cutoff after $4000 \mathrm{~cm}^{-1}$ is shown in Fig. 4. The spectacular strong peak around $3100 \mathrm{~cm}^{-1}$ disappears when the corresponding peak in $J_{0}(\omega)$ is discarded. We have verified that this peak and the precise value of the cutoff do not influence the reduced electronic dynamics. The spectral density $J_{1}(\omega)$ adopted for the simulations and the corresponding correlation function are shown in Fig. 4. $J_{1}(\omega)$ has been fitted by four Lorentzian functions. ${ }^{49}$ As expected, one observes a reduction of the system-bath coupling, in our case by about two orders of magnitude.

The memory kernel takes into account the coupling between the effective mode and the residual bath through the $C_{1}(t)$ correlation function.

$$
\begin{aligned}
& K(t-\tau) \rho_{S}(\tau) \\
& \quad=i\left[\mathbf{X}_{1},\left\{i C_{1}(t-\tau) e^{L_{S}(t-\tau)} \mathbf{X}_{1} \rho_{S}(\tau)\right\}+\{h . c .\}\right] .
\end{aligned}
$$



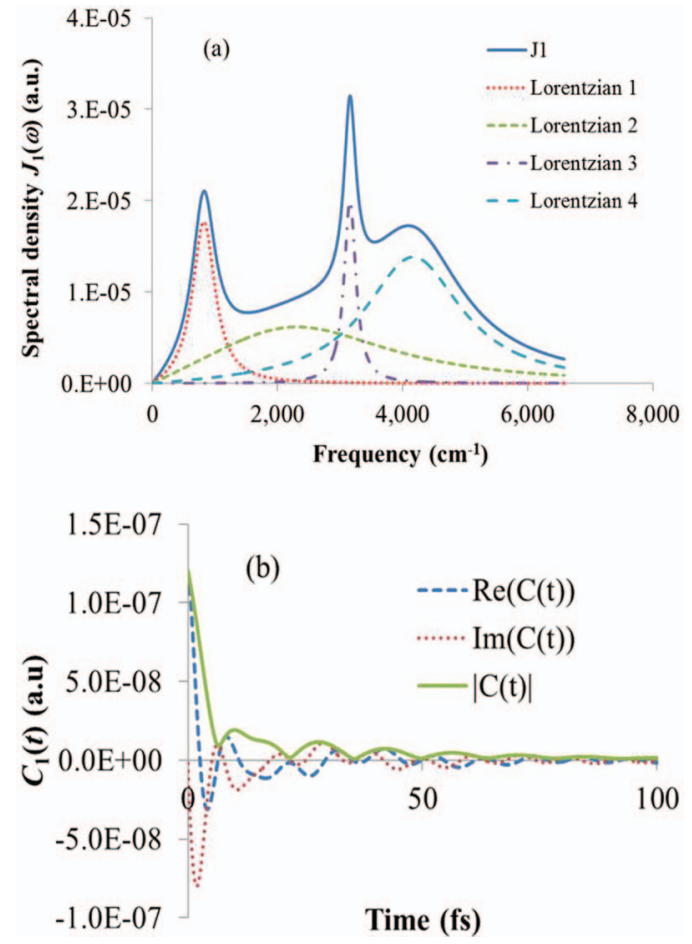

FIG. 4. (a) Fit of the residual spectral density $J_{1}(\omega)$ obtained by Eq. (15). (b) Corresponding bath correlation function [Eq. (3)] at $T=300 \mathrm{~K}$.

The auxiliary matrices are then defined by

$$
\rho_{1, k}(t)=i \alpha_{1, k} \int_{0}^{t} d \tau e^{i \gamma_{1, k}(t-\tau)} e^{L_{S}(t-\tau)} \mathbf{X}_{1} \rho_{S}(\tau) \quad k=1, n,
$$

and the second-order reduced equations in the effective-mode representation become

$$
\begin{aligned}
\dot{\rho}_{S}(t) & =L_{e f f} \rho_{S}(t)+i \sum_{k=1}^{n}\left[\mathbf{X}_{1}, \rho_{1, k}(t)+\rho_{1, k}^{\dagger}(t)\right], \\
\dot{\rho}_{1, k}(t) & =\left(i \gamma_{1, k}+L_{S}\right) \rho_{1, k}(t)+i \alpha_{1, k} \mathbf{X}_{1} \rho_{S}(t) \quad k=1, n .
\end{aligned}
$$

The system can be solved in a basis set with the Runge Kutta integrator $^{50}$ or in a grid representation by the Split Operator in the superoperator formalism by using the extension to non-adiabatic process. ${ }^{51}$

\section{RESULTS}

In the following, we discuss the results obtained for the spin-boson partitioning (Fig. 2(a)) and the effective-mode partitioning (Fig. 2(b)) in a comparative fashion.

\section{A. Spin-boson model}

From Fig. 3(b), one can see from the oscillatory pattern of $C_{0}(t)$ that the timescale of the bath dynamics is about $\tau_{\text {bath }}$ $\approx 30 \mathrm{fs}$. As shown in Table I, the Rabi period is small $\tau_{\text {Rabi }}$ $<\tau_{\text {bath }}$ except for the case of a large separation $R=3.5 \AA$ for which $\tau_{\text {Rabi }} \approx \tau_{\text {bath }}$. This situation corresponds to a slow bath. Therefore the Markov approximation is not relevant and
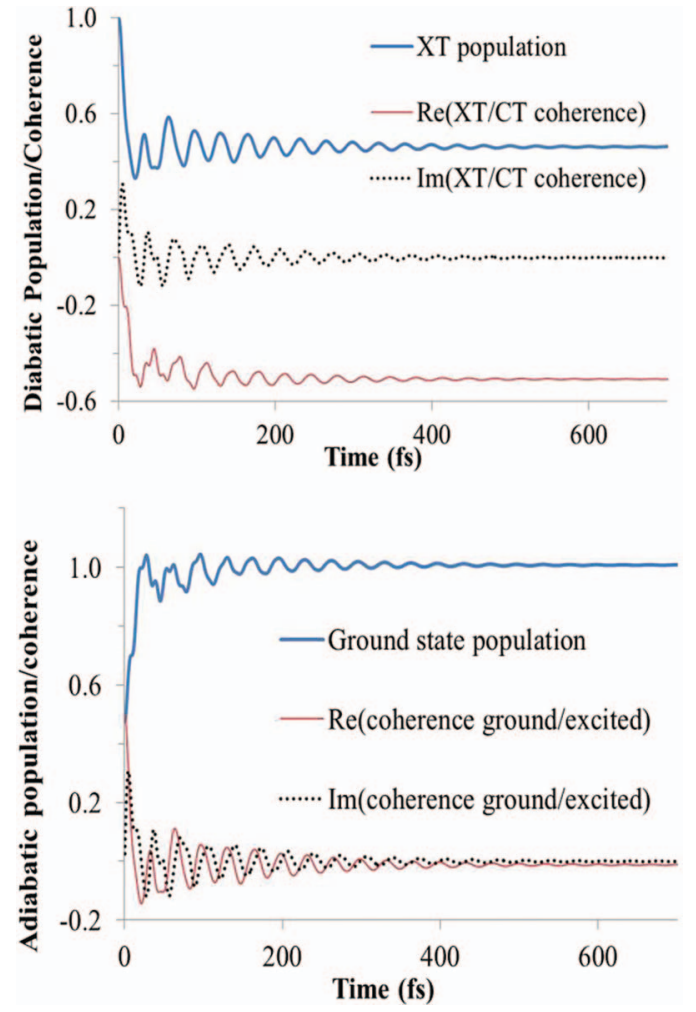

FIG. 5. Time evolving diabatic and adiabatic population and coherence for an inter-fragment distance $R=3.5 \AA$ in the spin-boson model. The initial state is the XT state. The correlation function at $T=300 \mathrm{~K}$ is given in Fig. 3.

it is not explored here. The bath dynamics is characterized by two parameters: the reorganization energy $\lambda$ related to the energy exchange between the electronic system and the oscillators and the bath frequency $\omega_{\text {bath }}=2 \pi / \tau_{\text {bath }}$. They can be gathered in a dimensionless parameter $\xi=\lambda / \hbar \omega_{\text {bath }}$. Here this coupling parameter is rather high $\xi=0.98$. The system is also characterized by a bias $\varepsilon=\Delta_{X T-C T} / V_{X T-C T}$ which is varying from $\varepsilon=2.5$ for $R=2.5 \AA$ to $\varepsilon=0.1$ for $R=3.5 \AA$. As already illustrated in Ref. 16, approximate methods give a poorer agreement with non-perturbative methods when the bias increases even in a moderate coupling regime. Our simulations with second-order equations fail leading to irrelevant values of $\operatorname{Tr}\left[\rho_{S}^{2}(t)\right]$ larger than 1 for all the studied inter-fragment distances except for $R=3.5 \AA$. For the given value of $\xi$, the only case giving acceptable results corresponds to a small bias $\varepsilon$, and a $\tau_{\text {Rabi }} \approx \tau_{\text {bath }}$. Figure 5 presents the population and electronic coherence evolution for this case which can be compared with the MCTDH dynamics in Sec. III C. Dissipation occurs from the first Rabi oscillation and one observes a relaxation towards the ground adiabatic state in about $200 \mathrm{fs}$. However, the population of this state slightly exceeds 1 as does $\operatorname{Tr}\left[\rho_{S}^{2}(t)\right]$ showing that the limit of validity of the perturbative approach is reached. The populations of the diabatic XT and CT states lead to 0.48 and 0.52 and the final coherence $\rho_{X T, C T}$ (given by its real part since the imaginary part vanishes) goes to the square root of their product, here about 0.5 . This shows that the final state is not a mixture of diabatic states but a superposition corresponding to the ground adiabatic state. In the adiabatic representation 
$\rho_{S}^{\text {adia }}=U^{\dagger} \rho_{S} U$, where $U$ diagonalizes the electronic Hamiltonian, the coherence effectively goes to zero since a single adiabatic state is populated.

\section{B. Effective mode representation}

Recasting the spin-boson Hamiltonian to include a single Brownian collective coordinate leads to a notable decrease of the system-bath coupling. In a case described by a single Lorentzian function $j_{k}(\omega)$, a relevant coupling parameter is the dimensionless ratio between the friction and the effective frequency $\eta / \Omega_{1}=2 \Gamma_{k} / \Omega_{1}$. For the main Lorentzian peak of $J_{0}(\omega)$, the ratio is about 0.15 thus more favorable for a perturbative treatment. In the case of a more structured spectral density, the coupling strength may also be evaluated by the ratio $\zeta=D_{0} / \Omega_{1}$ which is here equal to 0.08 a.u. The dimensionless parameter $\zeta^{2} / V_{X T-C T}$ used in Ref. 16 to discuss the quenching of the electronic coherence is also smaller than 1 varying from 0.03 for $R=2.5 \AA$ to 0.9 for $R=3.5 \AA$. Damped coherent oscillations are expected in all cases, with a faster damping in the case with a large inter-fragment distance and a small bias.

We first compare the results obtained in the spin-boson model and in the effective mode representation for the case $R$ $=3.5 \AA$. The effective potential energy curves and the correlation function are given in Figs. 2(b) and 4(b), respectively. The initial vibrational state is the ground state of the diabatic XT state. The corresponding energy is 0.0036 a.u. and thus this state is lying above the crossing point in this case. In this coordinate representation, the diabatic versus adiabatic states are coupled by potential couplings versus kinetic couplings, respectively. In order to analyze the electronic coherence and to disentangle the vibrational and electronic dynamics we define an electronic density matrix by tracing over the vibrational states of the effective mode:

$$
\rho_{e l}(t)=\operatorname{Tr}_{X_{1}}\left[\rho_{S}(t)\right]
$$

Fig. 6(a) shows the evolution of the population in the diabatic XT state (full blue line) and in the ground adiabatic state (dashed green line). The corresponding behavior obtained without dissipation is given in blue dashes-and-dots for the XT state, and in green dots for the adiabatic state. The damping of the Brownian coordinate $X_{1}$ occurs from the first oscillation. It is complete after $200 \mathrm{fs}$. The relaxation is faster than in the spin-boson model (see Fig. 5). One observes a trapping of the two wave packet components as revealed by the trajectories of the average position $\left\langle X_{1}(t)\right\rangle$ shown in Fig. 6(c). The average position in the CT state (red line) is about 5 a.u. and this value corresponds to the equilibrium position of the excited adiabatic state which has a final population $P=0.06$. Figure 6 (b) shows the real part and imaginary part of the electronic coherence in the diabatic or adiabatic representation (off-diagonal term of $\rho_{e l}(t)$ or $U^{\dagger} \rho_{e l}(t) U$, respectively). The imaginary parts are equal in both electronic representations and they vanish for $t \approx 200$ fs indicating that the population flux vanishes. ${ }^{52}$ Figure $6(\mathrm{~d})$ shows $\operatorname{Tr}\left[\rho_{e l}^{2}(t)\right]$ in different models. In the effective mode approach (full red line) one observes an ultrafast relaxation in about $20 \mathrm{fs}$. One
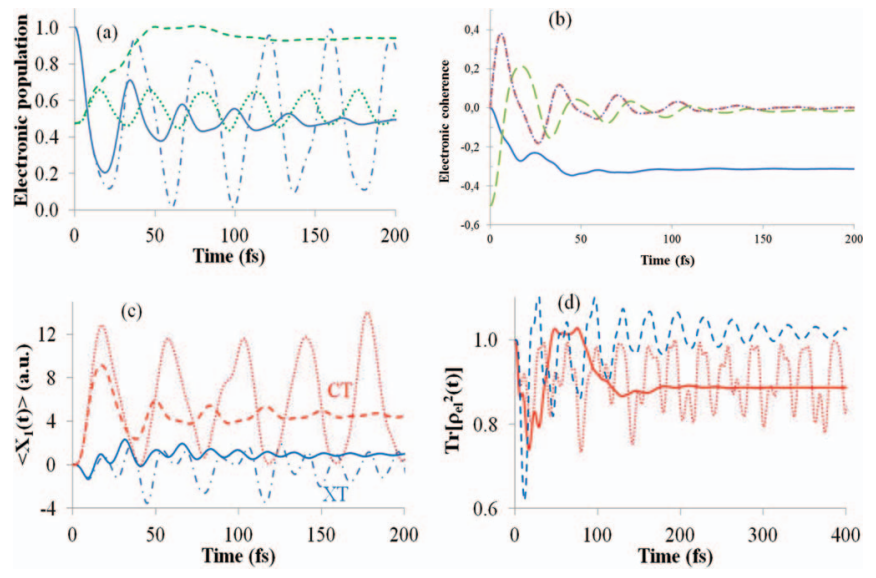

FIG. 6. Transfer dynamics in the effective mode model for $R=3.5 \AA$. (a) Population with dissipation, diabatic XT state (full blue line), ground adiabatic state (dashed green line); population without dissipation, XT state (blue dashed-dotted line), adiabatic state (green dots). (b) Diabatic coherence $\rho_{e l}(t)_{\mathrm{XT}, \mathrm{CT}}$, real part (full blue line) and imaginary part (red dashed line); adiabatic coherence $\left(U^{\dagger} \rho_{e l}(t) U\right)_{1,2}$, real part (dashed green line) and imaginary part (violet dots). (c) Trajectories of the average position $\left\langle X_{1}(t)\right\rangle$ with (full and dashed lines) and without (dashed-dotted line) dissipation, XT state in blue (curves at the bottom of the panel), and CT state in red (at the top). (d) Measure of the decoherence by $\operatorname{Tr}\left[\rho_{e l}^{2}(t)\right]$, red full line with bath dissipation, red dots without bath dissipation but dissipation due to $X_{1}$ and long violet dashes from the spin-boson model.

can see the role of the residual bath by comparing the full red line (full dissipation) with the dotted red line giving the effect of the effective mode only. The damping due to the secondary bath leads to an average value of the oscillations obtained with the primary mode only. The long-dot violet line is the spinboson result. One sees that the early decrease up to $10 \mathrm{fs}$ is well described by this crude model but following dynamics predicts too large oscillations in the trace which slightly exceeds 1 showing the limit of the method.

Next, we discuss the variation of the relaxation with the inter-fragment distance already addressed in previous works. ${ }^{2,53}$ It is expected that this intermolecular vibration is slow (the frequency is about $80 \mathrm{~cm}^{-1}$ ) and a sampling of different values of $R$ shows a strong modulation of the relaxation. As shown in Fig. 2, the diabatic curves are very different when $R$ decreases. One goes from the standard curve crossing for $R$ $=3.5 \AA$ to the Marcus inverted scheme with crossing on the left potential walls. The diabatic coupling $V_{\mathrm{XT}-\mathrm{CT}}$ increases by a factor 3 when the distance decreases from $3.5 \AA$ to $2.5 \AA$. The XT-CT energy gap $\Delta_{\text {XT-СT }}$ also increases by a factor 7 . The corresponding Rabi period decreases from about 30 fs to 6 fs. Figure 7(a) shows the evolving populations in the XT diabatic state. One clearly sees the different Rabi periods and the different limiting values of the XT population. The transfer is more complete for a short $R$ distance since the remaining population is varying from $P_{\mathrm{XT}}=0.48$ to $P_{\mathrm{XT}}=0.11$ but it is slower, passing from $200 \mathrm{fs}$ for $R=3.5 \AA$ to $1 \mathrm{ps}$ for $R=2.5 \AA$. The asymptotic values of $P_{\mathrm{XT}}$ match the weights of the XT state in the ground adiabatic state given in Table I (the successive values are $P_{\mathrm{XT}}=0.48,0.21,0.18,0.17$, and 0.11 from 3.5 to $2.5 \AA$ ). In the adiabatic view (see Fig. 7(b)), the limiting population in the ground state is about 0.95 . Figure 7(c) shows the evolution of the imaginary part of the 

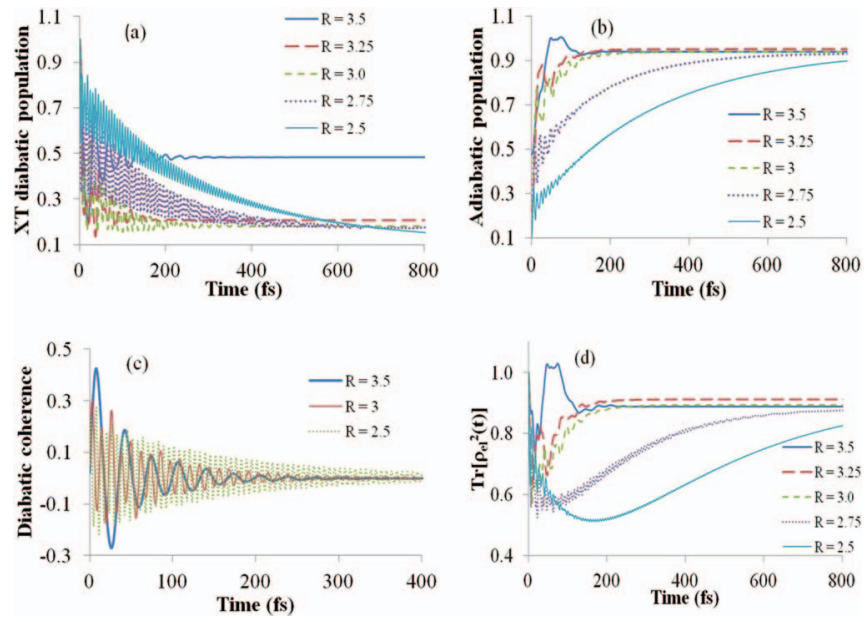

FIG. 7. Transfer dynamics in the effective mode model for a sampling of inter-fragment distances $R$ in $\AA$. (a) XT diabatic population. The initial condition is the ground vibrational state of the XT state. (b) Ground adiabatic state populations. (c) Imaginary part of the diabatic coherence for some values of $R$ only for clarity. The other $R$ values present a similar pattern. (d) Evolution of $\operatorname{Tr}\left[\rho_{e l}^{2}(t)\right]$.

diabatic coherence which is linked to the coherent population transfer. The imaginary part decays after 200 fs for $R=3.5 \AA$ to 1 ps for $R=2.5 \AA$. The real part, which is not presented here, evolves towards a constant indicating the formation of a superposed electronic state as discussed above for the $R=3.5 \AA$ case. In the adiabatic view, the real part decays toward a few percents only. The equilibrium state is thus a statistical mixing of adiabatic states but with a very small weight for the excited adiabatic state. This feature is also shown in $\operatorname{Tr}\left[\rho_{e l}^{2}(t)\right]$ which presents a specific profile with a fast decay followed by an increase toward a value smaller than 1 . The asymptotic value is 1 in the spin-boson model but here we obtain a small contamination by the excited state in the final mixture.

\section{Comparison with MCTDH}

Figure 8 compares the spin-boson and effective mode simulations with the $\mathrm{MCTDH}^{6}$ results obtained with 60 oscillators, using the same sampling for the spectral density $J_{0}(\omega)$ as in Ref. 2 and the same basis set. The MCTDH dynamics is carried out at $0 \mathrm{~K}$. The comparison addresses different points: initial decay, damping, and final equilibrium state. Panel (a) compares the three models for the case $R=3.5 \AA$ A. The initial decay rate of the XT diabatic state
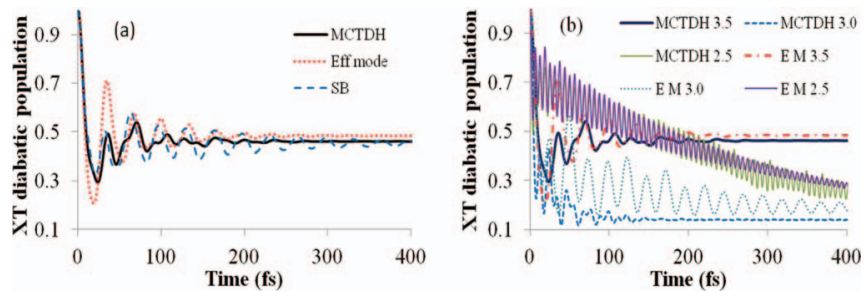

FIG. 8. Comparison of the population evolution obtained by MCTDH and by dissipative dynamics for three inter-fragment distances. (a) $R=3.5 \AA$. (b) $R=3.5 \AA$, $R=3.0 \AA$, and $R=2.5 \AA$.
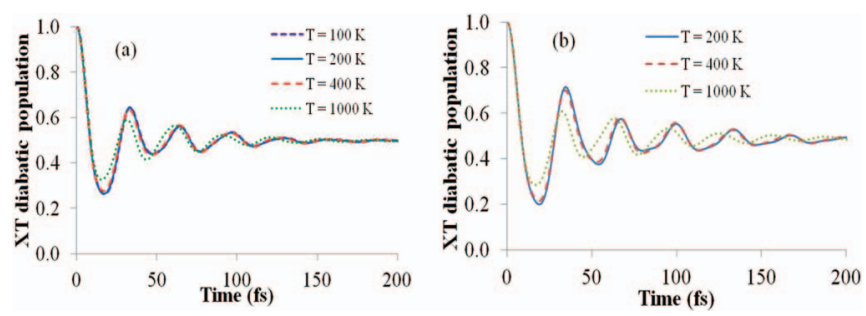

FIG. 9. Influence of the temperature on the dynamics for the case $R=3.5 \AA ̊$ A: (a) spin-boson model and (b) effective mode representation.

is identical in every approach. One observes that the early dynamics up to 25 fs gives similar oscillations reflecting the Rabi frequency. The damping is complete after about $200 \mathrm{fs}$ in the MCTDH simulation and in the effective mode model. The final equilibrium state obtained by the effective mode is close to the MCTDH value around 400 fs before the MCTDH recurrence occurring at the Poincaré period fixed by the discrete sampling (600 fs here). The spin-boson model predicts a too slow damping but a good asymptotic limit. However, the second order was questionable in this case. Panel (b) compares MCTDH and the effective mode for some inter-fragment distances. The Poincaré recurrence time is too small to reach equilibrium for $R=2.5 \AA$ Á and a new sampling with more oscillators would be necessary. In the other case, the comparison leads to the same conclusion, the effective mode model leads to a good estimate of the equilibrium state. The main difference comes from the damping which is sometimes slower in the effective mode representation, for instance for $\mathrm{R}=3.0 \AA$.

\section{Influence of the temperature on the dynamics}

Figure 9 shows that the temperature has a very small influence on the dynamics as suggested experimentally. ${ }^{54,55}$ The transfer leads to the same limit for the XT population in both models. The population evolution remains the same up to room temperature. As expected the amplitude of the Rabi oscillations diminishes only for very high temperature.

\section{CONCLUSION AND OUTLOOK}

The ultrafast electron transfer modelling an oligothiophene-fullerene junction calibrated by $a b$ initio quantum chemistry has been investigated by dissipative dynamics. The main features obtained by the previous MCTDH analysis $^{1,2}$ have been recovered. The early dynamics and the asymptotic values are well described. The real part of the coherence tends to a limiting value associated with a superposed state with similar diabatic XT and CT components. However the comparison remains qualitative for the damping rate.

The sampling of different values of the inter-fragment distance $R$ has revealed the high sensibility to the electronic coupling and energy gap on the transfer properties. When $R$ increases, the transfer is less complete but is faster.

From a methodological viewpoint, we have illustrated two points. First, the effective mode model is efficient to overcome some of the limitations of the simple spin-boson 
model described at the second order. The effective mode is extracted from the spin-boson spectral density computed from the displacements of the normal modes in both donor-acceptor electronic states. The decrease of the coupling to the residual bath lends validity to the perturbative approach which is not feasible for the full environment. Second, comparison with MCTDH confirms that dissipative dynamics is a powerful tool to describe relaxation at any temperature specifically for a long timescale. The MCTDH dynamics would require a sampling of initial conditions to represent a Boltzmann initial state. Besides, a long relaxation time of the order of the picosecond as in the short inter-fragment distance case should involve more modes to overcome the limitations imposed by the Poincaré recurrence time.

In a future work it is planned to analyze the efficiency of the hierarchical density matrix approach to go beyond the second-order approximation. Besides, it will be also very interesting to examine the role of the chain of effective modes beyond the truncation after the primary effective coordinate.

\section{ACKNOWLEDGMENTS}

We thank Dr. K. H. Hughes and Dr. Y. Justum for fruitful discussions.

${ }^{1}$ H. Tamura, I. Burghardt, and M. Tsukada, J. Phys. Chem. C 115, 10205 (2011).

${ }^{2}$ H. Tamura, R. Martinazzo, M. Ruckenbauer, and I. Burghardt, J. Chem. Phys. 137, 22A540 (2012)

${ }^{3}$ N. S. Sariciftci, L. Smilowitz, A. J. Heege, and F. Wudt, Science 258, 1474 (1992).

${ }^{4}$ C. J. Brabec, G. Zerza, G. Cerullo, S. De Silvestri, S. Luzzati, J. C. Hummelen, and S. Sariciftci, Chem. Phys. Lett. 340, 232 (2001).

${ }^{5}$ Y. Towada, T. Tsuneda, S. Yanagisawa, Y. Yanai, and K. A. Hirao, J. Chem. Phys. 120, 8425 (2004).

${ }^{6}$ H. D. Meyer, U. Manthe, and L. S. Cederbaum, Chem. Phys. Lett. 165, 73 (1990).

${ }^{7}$ U. Manthe, H. D. Meyer, and L. S. Cederbaum, J. Chem. Phys. 97, 3199 (1992).

${ }^{8}$ H. Tamura, E. R. Bittner, and I. Burghardt, J. Chem. Phys. 126, 021103 (2007); 127, 034706 (2007).

${ }^{9}$ R. Martinazzo, K. H. Hughes, F. Martelle, and I. Burghardt, Chem. Phys. 377, 21 (2010).

${ }^{10}$ K. H. Hughes, C. Christ, and I. Burghardt, J. Chem. Phys. 131, 024109 (2009).

${ }^{11}$ T. Westermann, R. Brodbeck, A. B. Rozhenko, W. Schroeller, and U. Manthe, J. Chem. Phys. 135, 184102 (2011).

${ }^{12}$ S. Nakajima, Prog. Theor. Phys. 20, 948 (1958).

${ }^{13}$ R. Zwanzig, Physica (Amsterdam) 30, 1109 (1964).

${ }^{14}$ C. Meier and D. Tannor, J. Chem. Phys. 111, 3365 (1999).

${ }^{15}$ A. Pomyalov, C. Meier, and D. Tannor, Chem. Phys. 370, 98 (2010).

${ }^{16}$ M. Thoss, H. Wang, and W. H. Miller, J. Chem. Phys. 115, 2991 (2001).

${ }^{17}$ J. Wu, F. Liu, Y. Shen, J. Cao, and R. J. Silbey, New J. Phys. 12, 105012 (2010).

${ }^{18}$ G. Dive, R. Robiette, A. Chenel, M. Ndong, C. Meier, and M. DesouterLecomte, Theor. Chem. Acc. 131, 1236 (2012).
${ }^{19}$ A. Chenel, G. Dive, C. Meier, and M. Desouter-Lecomte, J. Chem. Phys. A 116, 11273 (2012).

${ }^{20}$ U. Kleinekathöfer, J. Chem. Phys. 121, 2505 (2004).

${ }^{21}$ S. Welack, M. Schreiber, and U. Kleinekathöfer, J. Chem. Phys. 124, 044712 (2006).

${ }^{22}$ M. Schröder, U. Kleinekathöfer, and M. Schreiber, J. Chem. Phys. 124, 084903 (2006).

${ }^{23}$ Y. Tanimura and R. Kubo, J. Phys. Soc. Jpn. 58, 101 (1989).

${ }^{24}$ Y. Tanimura, J. Phys. Soc. Jpn. 75, 082001 (2006).

${ }^{25}$ J. Xu, R.-X. Xu, M. Luo, and Y. Yan, Chem. Phys. 370, 109 (2010).

${ }^{26}$ J. M. Moix and J. Cao, J. Chem. Phys. 139, 134106 (2013).

${ }^{27}$ M. Tanaka and Y. Tanimura, J. Chem. Phys. 132, 214502 (2010).

${ }^{28}$ A. Ishizaki, T. R. Calhoun, G. S. Schlau-Cohen, and G. R. Fleming, Phys. Chem. Chem. Phys. 12, 7319 (2010).

${ }^{29}$ J. Strümpfer and K. Schulten, J. Chem. Phys. 134, 095102 (2011).

${ }^{30}$ S. H. Yeh, J. Zhu, and S. Kais, J. Chem. Phys. 137, 084110 (2012).

${ }^{31}$ Y. Tanimura, J. Chem. Phys. 137, 22A550 (2012).

${ }^{32}$ I. Burghardt, R. Martinazzo, and K. H. Hughes, J. Chem. Phys. 137, 144107 (2012).

${ }^{33}$ K. H. Hughes, B. Cahier, R. Martinazzo, and I. Burghardt, "NonMarkovian reduced dynamics of ultrafast charge transfer at an oligothiophene-fullerene heterojunction," Chem. Phys. (submitted).

${ }^{34}$ A. Garg, J. N. Onuchic, and V. Ambegaokar, J. Chem. Phys. 83, 4491 (1985)

${ }^{35}$ E. Polak, J. Chem. Phys. 85, 865 (1986).

${ }^{36}$ A. M. Levine, M. Shapiro, and E. Polak, J. Chem. Phys. 88, 1959 (1988).

${ }^{37}$ V. Chernyak and P. Mukamel, J. Chem. Phys. 105, 4565 (1996).

${ }^{38}$ W. T. Pollard, A. K. Felts, and R. A. Friesner, Adv. Chem. Phys. 93, 77 (1996).

${ }^{39}$ J. Cao, J. Chem. Phys. 107, 3204 (1997).

${ }^{40}$ J. Cao and G. A. Voth, J. Chem. Phys. 106, 1769 (1997).

${ }^{41}$ A. Pereverzev, E. R. Bittner, and I. Burghardt, J. Chem. Phys. 131, 034104 (2009)

${ }^{42}$ L. S. Cederbaum, E. Gindensperger, and I. Burghardt, Phys. Rev. Lett. 94, 113003 (2005)

${ }^{43}$ E. Gindensperger, I. Burghardt, and L. S. Cederbaumn, J. Chem. Phys. 124, 144103 (2006)

${ }^{44}$ K. H. Hughes, C. D. Christ, and I. Burghardt, J. Chem. Phys. 131, 124108 (2009).

${ }^{45}$ R. Martinazzo, B. Vacchini, K. H. Hughes, and I. Burghardt, J. Chem. Phys. 134, 011101 (2011).

${ }^{46}$ R. Martinazzo, K. H. Hughes, and I. Burghardt, Phys. Rev. E 84, 030102 (2011).

${ }^{47}$ J. E. Subotnik, R. J. Cave, R. P. Steele, and N. Shenvi, J. Chem. Phys. 130, 234102 (2009).

${ }^{48}$ V. May and O. Kühn, Charge and Energy Transfer in Molecular System (Wiley-VCH, Berlin, 2011).

${ }^{49}$ See supplementary material at http://dx.doi.org/10.1063/1.4861853 for the parameters of the Lorentzian functions used in Figs. 2 and 4.

${ }^{50}$ W. H. Press, S. A. Teukolsky, W. T. Vetterling, and B. P. Flannery, Numerical Recipes in Fortran: The Art of Scientific Computing (Cambridge University Press, Cambridge, 1986).

${ }^{51}$ W. K. Lai, K.-A. Suominen, B. M. Garraway, and S. Stenholm, Phys. Rev. A 47, 4779 (1993).

${ }^{52}$ S. Mukamel, Principles of Nonlinear Optical Spectroscopy (Oxford University Press, New York, 1995).

${ }^{53}$ H. Tamura and M. Tsukada, Phys. Rev. B 85, 054301 (2012).

${ }^{54}$ R. D. Pensack, K. M. Banyas, and J. B. Asbury, J. Phys. Chem. B 114, 12242 (2010).

${ }^{55}$ R. D. Pensack, K. M. Banyas, and J. B. Asbury, J. Phys. Chem. Lett. 1, 2255 (2010) 